\title{
Agitation in patients with dementia: a systematic review of epidemiology and association with severity and course
}

\author{
Milena Anatchkova, ${ }^{1}$ Anne Brooks, ${ }^{1}$ Laura Swett, ${ }^{1}$ Ann Hartry, ${ }^{2}$ Ruth A. Duffy, ${ }^{3}$ \\ Ross A. Baker, ${ }^{3}$ Lene Hammer-Helmich, ${ }^{4}$ and Myrlene Sanon Aigbogun ${ }^{5}$ \\ ${ }^{1}$ Patient Centered Research, Evidera, Bethesda, MD, USA \\ ${ }^{2}$ Health Economics and Outcomes Research, Lundbeck LLC, Deerfield, IL, USA \\ ${ }^{3}$ Medical Affairs, Otsuka Pharmaceutical Development and Commercialization, Inc., Princeton, NF, USA \\ ${ }^{4}$ Real World Evidence and Epidemiology, H. Lundbeck A/S, Valby, Denmark \\ ${ }^{5}$ Health Economics and Outcomes Research, Otsuka Pharmaceutical Development and Commercialization, Inc., Princeton, NF, USA
}

ABSTRACT

Objectives: More than $90 \%$ of individuals with Alzheimer's disease (AD) experience behavioral and neuropsychiatric symptoms (NPS), such as agitation. However, little is known regarding the specific burden of agitation for Alzheimer's patients.

Design: A global systematic literature review was conducted in MEDLINE and Embase for studies of clinical, humanistic, and economic burden of agitation in AD/dementia published from 2006-2016. References of identified papers and related literature reviews were examined. Studies meeting predetermined inclusion criteria for burden of agitation/NPS were summarized.

Results: Eighty papers met the inclusion criteria for burden of agitation in dementia. Wide ranges of agitation prevalence were reported, but few papers provided information on incidence. The association of agitation with AD severity was presented in multiple studies; a few suggested positive association of agitation with mortality.

Conclusions: High prevalence of agitation is consistent with earlier reports, but several gaps in understanding of agitation in $\mathrm{AD}$ need further exploration.

Key words: agitation, Alzheimer's disease, systematic review, agitation burden

\section{Introduction}

Alzheimer's disease (AD) is the most common cause of dementia among older adults; it accounts for an estimated $60-80 \%$ of cases (Alzheimer's Association, 2015). In addition to loss of memory and cognition, more than $90 \%$ of individuals with $\mathrm{AD}$ experience behavioral and neuropsychiatric symptoms (NPS), such as agitation, aggression, depression, hallucinations, and delusions, over the course of their illness (Alzheimer's Society, 2011; Steinberg et al., 2008). NPS, also referred to as behavioral and psychological symptoms of dementia, are associated with increased morbidity, mortality, healthcare use, earlier nursing home placement, and caregiver

Correspondence should be addressed to: Myrlene Sanon Aigbogun, MPH, Health Economics and Outcomes Research, Otsuka Pharmaceutical Development \& Commercialization, Inc., 508 Carnegie Center, Princeton, NJ 08540, USA. Phone: +1.609.512.4456; Fax: +1.609.249.7342. Email: Myrlene.Sanon@, otsuka-us.com. Received 27 Mar 2018; revision requested 24 Jul 2018; revised version received 06 Sep 2018; accepted 29 Sep 2018. First published online 11 March 2019. burden and distress (Porsteinsson et al., 2014). While agitation is common among neuropsychiatric disorders, there was no consensus definition for it until recently-non-specific lay definitions were used (Cummings et al., 2015), making it difficult to compare studies and summarize existing evidence on the burden of agitation. Because of the lack of an accepted definition or assessment tool for agitation, there is a gap in the understanding of the burden of $\mathrm{AD}$ specific to agitation. A systematic review of the burden of agitation and NPS can help address this gap in knowledge.

The goal of this systematic literature review (SLR) was to map the existing evidence to better understand the total clinical, humanistic, and economic burden associated with agitation in adult patients with $\mathrm{AD}$. The specific objective of this paper is to describe the results regarding agitation incidence and prevalence, and its association with disease progression and mortality, in adult patients with $\mathrm{AD}$ based on results from the broader SLR. 
Table 1. PICOS criteria for burden of agitation in AD

\begin{tabular}{|c|c|c|}
\hline CRITERIA & INCLUSION & EXCLUSION \\
\hline Population & Alzheimer's disease, dementia with agitation & $\begin{array}{l}\text { Other pathology, pediatric population, non-human } \\
\text { studies, in-vitro studies }\end{array}$ \\
\hline Intervention & No limitations based on intervention & \\
\hline Comparator & No limitation based on comparator & \\
\hline Outcomes & $\begin{array}{l}\text { Agitation incidence, prevalence, mortality } \\
\text { associated with agitation, natural history } \\
\text { and agitation, disease severity }\end{array}$ & All others \\
\hline Study design & $\begin{array}{l}\text { Population observational studies } \\
\text { (e.g., cross-sectional, prospective cohort, } \\
\text { retrospective cohort), sample size }>50\end{array}$ & $\begin{array}{l}\text { Case study, study protocols, qualitative studies, } \\
\text { methodological studies, studies with sample } \\
\text { size }<50 \text {. Literature reviews retained for review } \\
\text { of references, but not summarized. }\end{array}$ \\
\hline Language & English & Non-English \\
\hline Time period & $2006-2016$ & Pre-2006 \\
\hline
\end{tabular}

Abbreviations: $\mathrm{AD}=$ Alzheimer's disease; PICOS = population, intervention, comparator, outcomes, study design.

\section{Patients and methods}

A systematic literature search, based on a preapproved Preferred Reporting Items of Systematic Reviews and Meta-Analyses (PRISMA) protocol and consistent with the PRISMA statement, was conducted for studies published between 2006 and 2016. The search was conducted in MEDLINE (via PubMed) and Embase. In addition, conference proceedings (including websites, posters, and meeting abstracts) from the two most recent meetings (last two years or last two major meetings) were searched for five professional organizations (Alzheimer's Association-International Conference on Alzheimer's Disease; International Psychogeriatric Association [IPA]; Alzheimer's Disease International [ADI]; American Association for Geriatric Psychiatry [AAGP]; International Society of Pharmacoeconomics and Outcomes Research [ISPOR]).

Blocks of medical subject heading (MeSH) terms were used to identify the most relevant articles, research papers, and conference papers that described the clinical burden associated with agitation in $\mathrm{AD}$. Agitation terms were based on the consensus definition of agitation issued by the IPA (Cummings et al., 2015), which includes behaviors that indicate severe emotional distress (e.g., irritability, rapid changes in mood), excessive motor activity (e.g., pacing, rocking, restlessness), physical aggression (e.g., pushing, hitting, kicking), and verbal aggression (e.g., shouting, cursing, yelling) - these behaviors must not be solely attributable to another psychiatric disorder (e.g., depression, psychosis). The focus of this review was specifically on agitation; however, studies that did not explicitly use the term agitation but reported on the behaviors noted above were included. Unless specifically stated otherwise, results are presented as a combination of agitation and related NPS in this report.
The search strategy used is presented in Table $S 1$ and Table S2 of the Supplementary Search Strategy Tables file and full list of references in presented in Supplementary Appendix, published as supplementary material online attached to the electronic version of this paper. SLRs were included in the initial search to review the reference list; this was to ensure all additional, relevant papers were included in the review.

All abstracts were reviewed using DistillerSR ${ }^{\circledR}$, a literature review extraction software, which assists with the organization, extraction, and categorization of the literature. Screening was performed by three trained reviewers at two levels. At Level 1, titles and abstracts of identified records were screened for exclusion criteria (Table 1). Thirty percent of the excluded abstracts were screened by a second, independent reviewer to ensure agreement. At Level 2, full-text articles were screened, and those meeting the eligibility criteria were tabled. The inclusion of all papers into the review was confirmed by a second reviewer. Data abstraction was completed in table format as reported. Qualitative synthesis of the information was conducted. No quantitative summaries were planned.

\section{Results}

The database search yielded 1,415 references, of which 1,407 remained after duplicates were removed. All 1,407 records were screened based on the protocol-defined criteria-365 were accepted for full-text screening. Of these, 206 papers were included in the final qualitative review of $\mathrm{AD}$ agitation burden (Figure 1). Of the 206 studies included in the final qualitative review, those providing information on burden of agitation were summarized by 


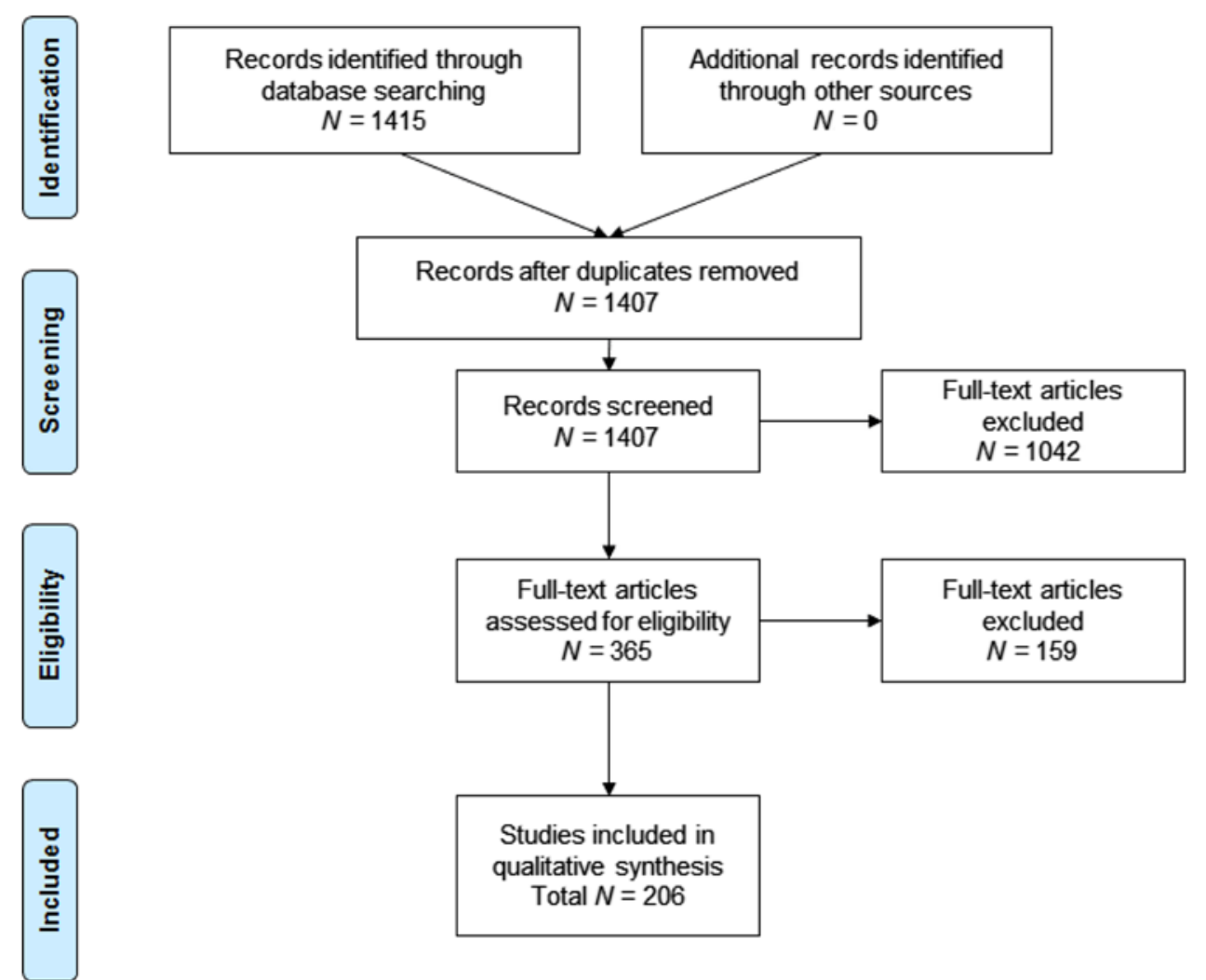

Figure 1. PRISMA flow diagram: SLR burden of illness associated with agitation in Alzheimer's disease.

protocol-defined groups. This paper summarizes findings related to incidence (4 papers), prevalence (55 papers), natural progression of agitation symptoms (17 papers), association of agitation and disease severity (34 papers and three conference abstracts), and association of agitation and mortality (5 studies). Publications related to humanistic, caregiver, and economic burden were also reviewed, but results are not reported in this paper (Anatchkova et al., 2017).

\section{Incidence of agitation/behavioral symptoms in AD}

Four studies evaluating the incidence of agitation/ behavioral symptoms in $\mathrm{AD}$ were identified; three from Norway and one from The Netherlands (Table 2). Incidence was defined in three studies as the proportion of participants who had no NPS at one timepoint but developed them at a later timepoint in the study. Cumulative incidence was defined as the proportion of patients who had no agitation/NPS at baseline, but developed these during the study. Of the three studies conducted in Norway, one identified the cumulative incidence of clinically significant agitation over a 2-year period to be $24.3 \%$ (Bergh et al., 2011), the 12-month cohort study found the incidence rate to be
$18.8 \%$ (Selbaek et al., 2008), and the 4-year longitudinal study indicated a cumulative incidence rate of 36\% (Selbaek et al., 2014). The Netherlands study, which was longitudinal, assessed 2-year incidence of agitation, which ranged from $10.9 \%$ to 18.2\% (Wetzels et al., 2010b). All studies used a population sample of nursing home $(\mathrm{NH})$ residents. Studies of incidence of agitation/behavioral symptoms in $\mathrm{AD}$ were only published in European countries; no studies of incidence were published in the North American, South American, or Asian regions. In addition, all studies presented findings for patients in nursing homes, while information on incidence of agitation for patients residing at home or seen as outpatients was lacking.

\section{Prevalence of agitation/behavioral symptoms in AD}

Fifty-five studies reported the prevalence of agitation and or behavioral symptoms in $\mathrm{AD}$ (Table 3). Study types included cross-sectional $(n=31)$, longitudinal or retrospective longitudinal $(n=23)$, and before/after study/interrupted time series $(n=1)$.

The overall prevalence of agitation ranged from $5 \%$ to $88 \%$ across all studies (Figure 2), with 21 (38\%) showing a prevalence of agitation $\geq 50 \%$. 


\begin{tabular}{|c|c|c|c|c|c|c|c|c|}
\hline CITATION & COUNTRY & $\begin{array}{l}\text { TYPE OF } \\
\text { STUDY }\end{array}$ & $\begin{array}{l}\text { TIME } \\
\text { PERIOD }\end{array}$ & $\begin{array}{l}\text { SAMPLE } \\
\text { SIZE }\end{array}$ & $\begin{array}{c}\text { AGE } \\
(\text { YEARS } \pm)\end{array}$ & $\begin{array}{c}\text { STUDY } \\
\text { POPULATION }\end{array}$ & SETTING & INCIDENCE \\
\hline $\begin{array}{l}\text { Bergh et al. } \\
\quad(2011)\end{array}$ & Norway & Cross-sectional & 2008-2010 & 169 & $84.9(6.7)$ & Dementia & $\mathrm{NH}$ & $\begin{array}{l}\text { Cumulative Incidence Rate } \\
24.2 \% \text { Agitation } \\
42.6 \% \text { Irritability } \\
82.9 \% \text { at least one NPS }\end{array}$ \\
\hline $\begin{array}{l}\text { Selbaek et al. } \\
\quad(2014)\end{array}$ & Norway & $\begin{array}{l}\text { Longitudinal } \\
\text { Cohort }\end{array}$ & 2004-2008 & 931 & $84.5(7.5)$ & Dementia & $\mathrm{NH}$ & $\begin{array}{l}\text { 12-month NPS Incidence Rates } \\
47.3 \% \text { Any symptom } \\
18.5 \% \text { Agitation } \\
22.7 \% \text { Irritability } \\
13.3 \% \text { Aberrant motor behavior } \\
\text { 4-year Cumulative Incidence Rates } \\
36.0 \% \text { Agitation } \\
51.7 \% \text { Irritability }\end{array}$ \\
\hline $\begin{array}{l}\text { Wetzels et al. } \\
\text { (2010a) }\end{array}$ & Netherlands & Longitudinal & $\begin{array}{c}2 \text { years } \\
\text { (specific years NR) }\end{array}$ & 117 & $81.7(7.4)$ & Dementia & $\mathrm{NH}$ & $\frac{\text { Incidence Rate (2-year range) }}{(10.9 \%-18.2 \%)}$ \\
\hline $\begin{array}{l}\text { Selbaek et al. } \\
\text { (2008) }\end{array}$ & Norway & Cohort & $\begin{array}{c}1 \text { year } \\
2004 / 2005-2005 / 2006\end{array}$ & 923 & NR & Dementia & $\mathrm{NH}$ & $\begin{array}{l}\text { 12-month NPS Incidence Rates } \\
43.8 \% \text { Any symptom } \\
18.8 \% \text { Agitation } \\
23.0 \% \text { Irritability } \\
13.8 \% \text { Aberrant motor behavior }\end{array}$ \\
\hline
\end{tabular}

Abbreviations: $\mathrm{NH}=$ nursing home; NPS = neuropsychiatric symptoms; NR = not reported. 
Table 3. Agitation studies reporting prevalence, natural progression, and association with AD severity

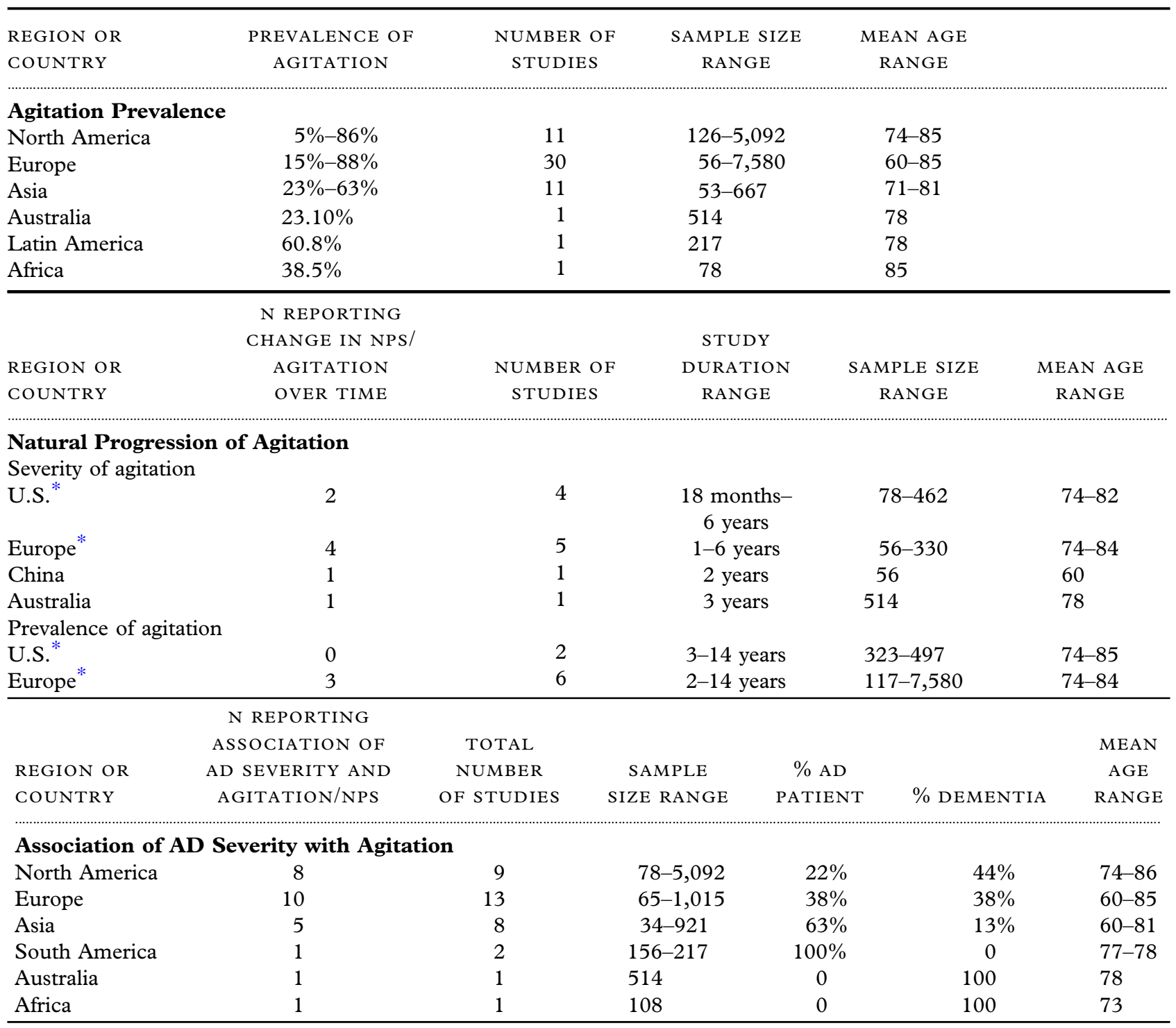

* One study U.S./Europe.

Abbreviations: $\mathrm{AD}=$ Alzheimer's disease; NPS = neuropsychiatric symptoms; U.S. $=$ United States.

Twenty-three of the 55 studies (42\%) reported prevalence of at least one neuropsychological symptom and reported a range of $40 \%$ to $100 \%$. Community-dwelling or outpatient clinic participants were the primary population studied in 26 $(47 \%)$ studies; $\mathrm{NH}$ or group home residents were the primary population in $16(29 \%)$ studies; a combination of $\mathrm{NH}$ and community-dwelling participants were the primary population in five $(9 \%)$ studies; hospital patients were the primary population in five $(9 \%)$ studies, and three (5\%) studies did not report the source of their primary population. Most studies screened participants only for $\mathrm{AD}$ $(n=28)$ or dementia $(n=25)$, while a couple screened for memory loss/cognitive impairment $(n=2)$.

\section{Prevalence variations by geographical region}

Europe

Participants specifically included in the 30 studies $(55 \%)$ that originated from Europe included participants with $\mathrm{AD}(n=14)$ and dementia $(n=15)$; one study recruited for cognitive impairment, looking at NPS prevalence rates (Gustafsson et al., 2013). Of these 30 studies, 15 were cross-sectional (including one retrospective cross-sectional study) and 15 were longitudinal. The range of prevalence of agitation was reported to be $24 \%$ to $88 \%$, with 14 studies (47\%) showing an agitation prevalence rate $\geq 50 \%$. Sixteen studies $(53 \%)$ reported prevalence rates of at least one NPS, which ranged from $49.6 \%$ to $96.1 \%$. Prevalence rates for agitation in European 


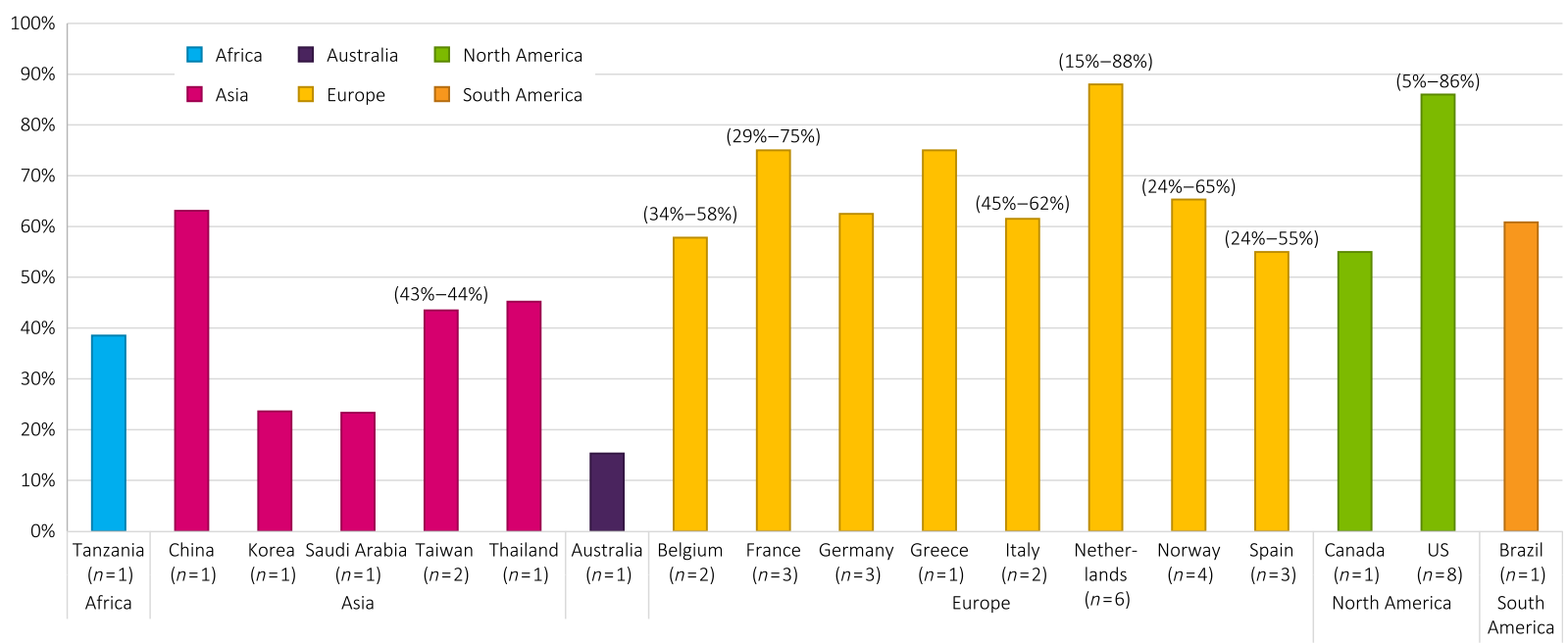

Figure 2. Prevalence of agitation by geographic region. Abbreviation: U.S. = United States.

studies ranged from $24 \%$ to $88 \%$ for $\mathrm{NH}$ or geriatric facility participants $(n=13) ; 18.6 \%$ to $76 \%$ for community-dwelling participants $(n=13) ; 34.1 \%$ to $75.4 \%$ for $\mathrm{NH}$ plus community-dwelling participants $(n=2)$; and one study reported a prevalence rate for hospital participants of $44.8 \%$ (D'Onofrio et al., 2012).

Asia

Participants in the 11 studies $(20 \%)$ that originated from Asia included participants with $\operatorname{AD}(n=7)$ and dementia $(n=4)$. The range of prevalence of agitation was reported as $23.3 \%$ to $78 \%$ in 10 studies, with two $(17 \%)$ showing an agitation prevalence rate $\geq 50 \%$. Four studies (33\%) reported prevalence rates of at least one NPS, which ranged from $74.9 \%$ to $100 \%$. Prevalence rates for agitation ranged from $23.3 \%$ to $63.1 \%$ for community-dwelling or outpatient participants $(n=7) ; 16.2 \%$ to $52.2 \%$ for $\mathrm{NH}$ plus community-dwelling participants $(n=3)$; and one study reported a prevalence rate for hospital participants of $52 \%$ for women and $78 \%$ for men (Kitamura et al., 2012).

\section{North AMERICA}

Participants in the 11 studies $(20 \%)$ that originated from North America (nine in the United States [U.S.]) included participants with $\mathrm{AD}(n=4)$, dementia $(n=4)$, the general geriatric population $(n=2)$, and participants with memory loss $(n=1)$. The range of prevalence of agitation was reported as $5 \%$ to $86 \%$ in 10 studies, with four $(36 \%)$ showing an agitation prevalence rate $\geq 50 \%$. Two studies (18\%) reported prevalence rates of at least one NPS, which ranged from $50.9 \%$ to $89 \%$. Prevalence rates for agitation ranged from: $6.9 \%$ to $86 \%$ for community-dwelling participants $(n=5), 50.4 \%$ for $\mathrm{NH}$ participants $(n=1)$, and $31 \%$ for $\mathrm{NH}$ plus community-dwelling participants $(n=1)$ (Orengo et al., 2008); three studies that did not report the source of their study sample reported a prevalence rate ranging from $5.3 \%$ to $40 \%$.

Other Countries

Participants in the three studies (5\%) that originated from other countries (Australia, Tanzania, and Brazil) included participants with $\mathrm{AD}(n=1)$ and dementia $(n=2)$. The range of reported prevalence of agitation/aggressiveness was $15.3 \%$ to $60.8 \%$, with one study (33\%) showing an agitation prevalence rate $\geq 50 \%$. Two studies $(67 \%)$ reported prevalence rates of at least one NPS, which ranged from $88.4 \%$ to $98 \%$.

\section{Natural progression of agitation/behavioral symptoms in AD}

Seventeen studies that evaluated disease progression of agitation/behavioral symptoms in $\mathrm{AD}$ were identified. Studies presenting results on changes in severity of NPS or changes in prevalence rates over time were reviewed.

Of the 10 papers that evaluated changes in severity/frequency of agitation/NPS over time, six reported an increase (Brodaty et al., 2015; Pan et al., 2013; Selbaek et al., 2008; Trzepacz et al., 2013; Vogel et al., 2015; Zahodne et al., 2015); one reported a decrease (Wolf-Ostermann et al., 2012); and one reported no change (Bergh et al., 2011). Two studies had mixed results. Burgio and colleagues (2007) reported little change in agitation over an 18-month period according to staff report; while direct observation showed a statistically significant $(P$ value $<0.05)$ trajectory of agitation with decreasing trend. In the study conducted by Fauth and colleagues (2006), using a mixed-model analyses, group-level analysis showed no change, while 
intra-individual results suggested an increase in prevalence of disruptive behavior. Evaluated timeframes ranged from 24 weeks to 6 years. The evidence suggests there is an increase in severity of agitation symptoms over time, although some studies provided mixed results.

Of the nine papers evaluating changes in prevalence of agitation/NPS over time, six reported an increase (Gonfrier et al., 2012; Lustenberger et al., 2011; Scarmeas et al., 2007; Selbaek et al., 2008, 2014; Wetzels et al., 2010b); two reported no or little change (Bergh et al., 2011; Mitchell et al., 2009); and none reported a decrease. Of note, agitation persisted at most timepoints over 4 years in the study by Hendriks and colleagues (2015) but decreased in the last week of life. Evaluated timeframes ranged from 1 to 14 years. The evidence suggests agitation/ NPS becomes more prevalent over time.

The most common clinical outcome assessment (COA) used in the studies was the Neuropsychiatric Inventory (NPI). Of the studies that evaluated the change in overall NPS over time $(n=14), 11$ used the NPI as the primary endpoint measure. Other measures used included the Cohen-Mansfield Agitation Inventory (CMAI), the Disruptive Behavioral Symptoms measure (DBS), nurse or staff reports, direct observation, and the Columbia University Scale for Psychopathology in AD (CUSPAD). This variation in assessment methods can contribute to the variations in reported results.

\section{Variations in natural progression by geographical region}

Nearly half of the studies on natural progression were completed in Europe $(n=9 / 17)$; four studies were completed in the United States, one in Asia, and one in Australia, and two were a combined effort between Europe and the U.S. A greater percentage of European studies used a population sample of $\mathrm{NH}$ residents than U.S. studies $(78 \%$ vs. $50 \%$ ), and a greater percentage of U.S. studies used a population sample of community-dwelling residents than European studies (50\% vs. $22 \%$ ); the Asian study used a hospital population (Pan et al., 2013) and the Australian study used a communitydwelling population (Brodaty et al., 2015).

There were noteworthy differences in results of the course of agitation over time between the United States and Europe. More European studies showed an increase in agitation prevalence over time than U.S. studies ( $67 \%$ vs. $25 \%$ ), and more U.S. studies showed little or no change in prevalence or frequency over time than European studies (50\% vs. $11 \%)$. One European study indicated no change in agitation severity (Bergh et al., 2011), and one study showed mixed results- the NPI total score decreased over time but all domains of the CMAI showed an increase in agitation over time (WolfOstermann et al., 2012). Of the combined U.S./ European studies, mixed results were reported; one showed an increase in disruptive behavioral symptoms over time (Scarmeas et al., 2007), and the other demonstrated that the prevalence of agitation worsened over time, but was not significant when adjusting for cognitive decline (Zahodne et al., 2015). Both the Asian and Australian studies showed an overall increase in NPS severity (Brodaty et al., 2015; Pan et al., 2013), although only one found increases in agitation outcomes specifically. (Brodaty et al., 2015)

\section{Disease severity and agitation/behavioral symptoms in AD}

Thirty-four studies assessed some association between $\mathrm{AD}$ severity and NPS/agitation. Of these, $25(74 \%)$ established an association between NPS or agitation and disease severity, four (12\%) established a trend towards an association between the two without establishing statistical significance or testing for it, one study (3\%) reported mixed results, and four studies (12\%) reported no association between agitation/NPS and AD.

Results were examined separately for studies that explicitly noted assessment of agitation and those that assessed related NPS, but did not focus explicitly on agitation. Of the 34 studies that investigated the relationship between disease severity and NPS, $25(74 \%)$ evaluated the relationship between agitation and disease severity, nine studies (26\%) evaluated the relationship between NPS and disease severity, and six studies (18\%) evaluated both agitation and overall NPS by disease severity.

Of the 25 studies that evaluated the relationship between agitation and disease severity, 17 (68\%) demonstrated a significant association between the two, four studies (16\%) showed a trending association (de Oliveira et al., 2015; Ismail et al., 2007; Karttunen et al., 2011; Park et al., 2015), and four studies (Fernandez-Martinez et al., 2010; Kim and Lee, 2014; Pinidbunjerdkool et al., 2014; Treiber et al., 2008) (16\%) demonstrated that there was no significant relationship between agitation and disease severity.

Of the 17 studies that showed a significant relationship between agitation and disease severity, 11 $(65 \%)$ were cross-sectional and six (35\%) were longitudinal. Dementia severity in these 17 studies was evaluated by the Mini-Mental State Examination (MMSE) $(n=4)$ (Burgio et al., 2007; Hamuro et al., 2007; Kasai et al., 2015; O'Donnell et al., 2007), the Global Deterioration Scale (GDS) $(n=2)$ (Mulders et al., 2016; Steinberg et al., 2006), the 
Clinical Dementia Rating Scale (CDR; $n=4$ ) (Helvik et al., 2016; Liu et al., 2007; Selbaek et al., 2014; Tanaka et al., 2015), both the MMSE and CDR $(n=2)$ (D'Onofrio et al., 2012; Peters et al., 2015), or studies did not specify $(n=5)$. Of these 17 studies showing significant results, agitation was reported as being measured by the NPI in four studies (Fernandez Martinez et al., 2008; Helvik et al., 2016; Peters et al., 2015; Tanaka et al., 2015), the CMAI in two studies (Mulders et al., 2016; Steinberg et al., 2008), and Behavioral Pathology in Alzheimer's Disease Rating Scale (BEHAVE-AD) in one (Liu et al., 2007). Other studies either did not specify or used a study-unique definition of agitation $(n=10)$.

Of the 11 cross-sectional studies $(n=11 / 17)$ that reported a significant relationship between agitation and disease severity, three used the CDR to define dementia severity and one study showed agitation to be significantly predictive of the severity of cognitive impairment (moderate-severe dementia: $\mathrm{CDR}=2-3$ and MMSE score $<18$ ) in AD patients (D'Onofrio et al., 2012). A second study, by Helvik and colleagues (2016), showed that some CDR groups (defined as $\mathrm{CDR}<1$ : none; $\mathrm{CDR}=1$ : mild; $\mathrm{CDR}=2$ : moderate, $\mathrm{CDR}=3$ : severe) were significantly associated with agitation. Tanaka and colleagues (2015) compared NPI agitation scores by CDR groups (mild, moderate, and severe classifications); the association was significant between agitation and all CDR groups ( $P$ value $<0.001)$.

Six longitudinal studies demonstrated significant association between agitation and disease severity. One study reported disease severity using CDR scores (Selbaek et al., 2014), showing that CDR scores of $2(P$ value $=0.02)$ or $3(P$ value $<0.001)$ were significantly predictive of agitation. Another study compared GDS groups (defined as 1, 2, 3, 4, 5, 6, and 7) against CMAI subdomains, demonstrating that agitation was significantly predictive of dementia severity in all CMAI subdomains (Steinberg et al., 2008). A third study, by Morgan and colleagues (2013), communicated a significant association between agitation and dementia severity $(\mathrm{r}=-0.19, P$ value $<0.001)$. Another study compared $\mathrm{AD}$ groups by mild, moderate, and severe $\mathrm{AD}$, demonstrating that mild vs. moderate $\mathrm{AD}$ groups showed significantly significant differences in agitation levels ( $P$ value $=0.045$ ) (Pink et al., 2015). A study by Peters and colleagues (2015) demonstrated that agitation was significantly predictive of progression to severe dementia (hazard ratio: $2.946, P$ value $=0.004)$. Severe dementia was measured using the MMSE and CDR; severe AD was defined as an MMSE score of $\leq 10$ or CDR score $=3$. The sixth study reported that the severity of dementia increased the odds of agitation (odds ratio: 2.42 , confidence interval: $1.81-3.23, P$ value $<0.01$ ) (Steinberg et al., 2006).

\section{Papers investigating the association between AD severity and other NPS}

Nine studies $(n=9 / 34)$ evaluated the relationship between NPS and disease severity, either using a total score for NPS or by evaluating the relationship by NPS type. Additionally, five studies that evaluated the relationship between agitation and disease severity also included results on other NPS, for a total of 14 studies. Of these 14 studies, 10 (Amoo et al., 2011; Brodaty et al., 2015; Conde-Sala et al., 2016; D'Onofrio et al., 2012; 2016; Karttunen et al., 2011; Khoo et al., 2013; Koppitz et al., 2015; Spalletta et al., 2010; Stella et al., 2016) demonstrated a statistically significant relationship between NPS and disease severity. Four (de Oliveira et al., 2015; Ismail et al., 2007; Karttunen et al., 2011; Park et al., 2015) did not. Eight of the 10 statistically significant studies demonstrated significant associations between the degree of dementia severity and NPS, measured by total NPS score (Brodaty et al., 2015; Conde-Sala et al., 2016; D'Onofrio et al., 2012; Karttunen et al., 2011; Khoo et al., 2013; Koppitz et al., 2015; Spalletta et al., 2010; Stella et al., 2016). One study showed a significant relationship between specific NPS type and disease severity, including disinhibition and aberrant motor behavior (Amoo et al., 2011); another study, measuring both general and specific NPS, found a significant relationship between specific NPS type and disease severity, including irritability/lability and aberrant motor activity (D'Onofrio et al., 2016).

\section{Regional differences in associations of disease severity with agitation}

Most studies evaluating the relationship between agitation/NPS and disease severity originated from Europe $(n=13 / 34)$; followed by North America $(n=9 / 34)$, Asia $(n=8 / 34)$, South America $(n=2 /$ $34)$, Africa $(n=1 / 34)$, and Australia $(n=1 / 34)$. Noteworthy regional differences included differences in population sample by region and residence of study population by region.

Studies originating in South America and Africa had the most studies that identified $\mathrm{AD}$ patients as their study population $(100 \%)$, followed by Asia $(63 \%, n=5$; Dementia $=12 \%, n=1$; Mixed $=25 \%, n=2)$, Europe (38\%, $n=5$; Dementia $=38 \%, n=5$; Mixed $=23 \%, n=3$ ), and North America $(22 \%, n=2$; Dementia $=44 \%, n=5$; Memory loss $=22 \%, n=2$ ).

The most common place of residence from which participants were drawn was communitydwelling residents, and the highest sample draw of 
community-dwelling residents came from South American studies $(100 \%, n=2)$, followed by Asian $(63 \%, n=5)$, North American $(63 \%, n=5)$, and European $(46 \%, n=6)$. NH resident study populations were more common in European $(46 \%, n=6)$ and North American studies $(25 \%, n=2)$ than Asian $(22 \%, n=2)$ or South American $(0 \%, n=0)$.

Significant findings associated with agitation/ NPS and disease severity were found more often in studies originating in North America (89\%, $n=8)$, followed by Europe $(77 \%, n=10)$, Asia $(63 \%, n=5)$, and South America $(50 \%, n=1)$. The single studies from Australia and Africa also reported significant association. Non-significant findings of this association were most common in Asia $(22 \%, n=2$; trending findings $11 \%, n=1)$, followed by European studies $(8 \%, n=1$; trending findings $8 \%, n=1)$. Two regions had no nonsignificant results, but had one study each with trending findings (South America $n=1,50 \%$; North America $n=1,11 \%$ ).

\section{Mortality associated with agitation in AD}

There were five studies that provided information on the association between agitation and mortality, and issues around death, which were included in the review (Table 4). The studies were from different settings, including nursing homes $(n=2)$, an outpatient clinic $(n=1)$, and mixed settings $(n=1)$; one setting was not reported.

Most studies provided estimates of association of agitation with mortality $(n=3)$. Two studies (Peters and colleagues, 2015; Sampson and colleagues, 2015) reported a significant association of agitation-related symptoms and mortality. Peters and colleagues (2015) reported results of a longitudinal population-based study, using data from 1995-2009, and reported that agitation is a significant predictor of death, controlling for age of dementia onset, gender, education level, general health rating, and apolipoprotein $\mathrm{E}$ epsilon 4 (APOE- $\varepsilon 4$ ) genotype status. Results of a longitudinal cohort study of people with dementia admitted to an acute care hospital also reported significant association between severity of NPS and mortality. (Sampson et al., 2015) In a retrospective cohort study with $\mathrm{NH}$ $\mathrm{AD}$ patients, while reduction in behavioral symptoms as a result of treatment was associated with reduced risk of mortality, this association did not reach statistical significance. (Huang et al., 2015)

The study by Koyama and colleagues (2015) did not directly assess the association between mortality and agitation in dementia patients. Instead, it provided information on the relationship between suicidal ideation of dementia patients and NPS. Ten percent of the dementia patients in the sample had suicidal thoughts. NPI-Agitation/Aggression (NPIA/A) scores were statistically different for patients with and without suicidal thoughts $(P$ value $=0.004)$

The Vandervoort and colleagues (2013) study investigated palliative quality of life in dementia patients. Patients that were included in this research were patients that had died in the previous 3 months. In the last month of life, pain, fear, anxiety, resistance to care, and agitation were the most frequently reported symptoms of dementia.

A few studies directly examined the association between agitation in dementia and mortality risk, and provided some evidence supporting a positive relationship. However, there is minimal research in this area. Further research exploring the relationship between dementia agitation and risk of death, and providing an understanding of the interaction of that relationship, would be beneficial.

\section{Discussion}

In this systematic review, a wide range of agitation prevalence rates were reported ( $5 \%$ to $88 \%$ ), with rates varying somewhat by geographic region (lower ranges were reported for Asia). Most studies, however, reported prevalence rates that were under $50 \%$. Few estimates were based on large samples. The years for which estimates were provided varied, patients from both outpatient and clinical settings were used, and many agitation assessments were used. These variations in study design make it difficult to provide a reliable estimate on the true prevalence rate of agitation symptoms in $\mathrm{AD}$ patients.

Seventeen studies reported on the natural progression of agitation/NPS in AD. Results suggested agitation severity ratings increase over time, while the proportion of patients with symptoms increase slightly or remain stable over time. While some geographic variations were detected in prevalence rates and the course of agitation and associations reported between $\mathrm{AD}$ severity and agitation, the heterogeneity of the included studies on multiple dimensions suggests that such differences should be examined tentatively and with caution.

The relationship between $\mathrm{AD}$ severity and agitation was evaluated in a substantial number of studies and was supported by most of them, providing one of the most robust findings of the review.

Overall, this review was broad in scope, and the results on agitation prevalence align with findings from earlier reviews that focused on prevalence and progression of NPS in patients with AD and/or dementia. Borsje and colleagues (2015) conducted a systematic review of studies reporting the course of NPS in community-dwelling adults with dementia, 
Table 4. Studies on agitation and mortality

\begin{tabular}{|c|c|c|c|c|c|c|c|c|}
\hline CITATION & COUNTRY & $\begin{array}{c}\text { TYPE } \\
\text { OF STUDY }\end{array}$ & TIME PERIOD & $\begin{array}{l}\text { SAMPLE } \\
\text { SIZE }\end{array}$ & AGE & $\begin{array}{c}\text { STUDY } \\
\text { POPULATION }\end{array}$ & SETTING & $\begin{array}{c}\text { SURVIVAL/RISK } \\
\text { OF DEATH }\end{array}$ \\
\hline $\begin{array}{l}\text { Huang et al. } \\
\text { (2015) }\end{array}$ & US & $\begin{array}{l}\text { Retrospective } \\
\text { Longitudinal }\end{array}$ & $\begin{array}{l}\text { Running baseline/ } \\
\text { reassessment } \\
2006-2009\end{array}$ & 3,696 & $\begin{array}{l}83.2(9.8) \\
\text { Anti-psychotic } \\
82.3(10.6) \\
\text { Anti-depressant }\end{array}$ & Dementia & $\mathrm{NH}$ & $\begin{array}{l}\text { Patients on APs whose symptoms } \\
\text { improved less likely to die with } \\
6 \text { months, but effect NS: } \\
\text { LR 6-month mortality OR } \\
(95 \% \mathrm{CI})=0.89(0.70-1.13) \text {; } \\
\text { Cox mortality HR (adjusted) } \\
(95 \% \mathrm{CI})=0.93(0.81-1.07)\end{array}$ \\
\hline $\begin{array}{l}\text { Peters et al. } \\
\quad(2015)\end{array}$ & US & Longitudinal & 1995-2009 & $\begin{array}{l}5,092(335 \\
\mathrm{AD} \text { cases })\end{array}$ & $84.3(6.4)$ & $\begin{array}{l}\text { Population } \\
\text { sample }\end{array}$ & NR & $\begin{array}{l}\text { Earlier death related to affective } \\
\text { and mild NPS }(\mathrm{HR}=1.951 \\
\mathrm{p}=<0.001)\end{array}$ \\
\hline $\begin{array}{l}\text { Vandervoort } \\
\text { et al. (2013) }\end{array}$ & Belgium & Post-mortem & 2010 & 198 & $\begin{array}{l}86.7 \text { age at } \\
\text { death (SD NR) }\end{array}$ & Dementia & $\mathrm{NH}$ & $\begin{array}{l}\text { Agitation among the top } 3 \\
\text { distressing symptoms during } \\
\text { the last months of life. }\end{array}$ \\
\hline $\begin{array}{l}\text { Koyama } \\
\quad \text { et al. }(2015)\end{array}$ & Japan & Cross-sectional & 2007-2013 & 634 & 77.3 (SD NR) & $\begin{array}{l}\text { Dementia } \\
\text { and } \mathrm{AD}\end{array}$ & Outpatient & $\begin{array}{l}10.1 \% \text { prevalence of suicidal ideation } \\
\text { NPI-A/A statistically different } \\
\text { for patients with and without } \\
\text { ideation }(\mathrm{p}=0.004)\end{array}$ \\
\hline $\begin{array}{l}\text { Sampson } \\
\text { et al. }(2014)\end{array}$ & UK & $\begin{array}{l}\text { Longitudinal } \\
\text { cohort }\end{array}$ & 2011-2012 & 230 & $87.2(5.9)$ & Dementia & Mixed & $\begin{array}{l}\text { Severity of BEHAVE-AD } \\
\text { aggression as predictor of mortality: } \\
\text { OR } 1.23 ; 95 \% \text { CI } 1.06-1.44 ; \mathrm{p}=0.008\end{array}$ \\
\hline
\end{tabular}

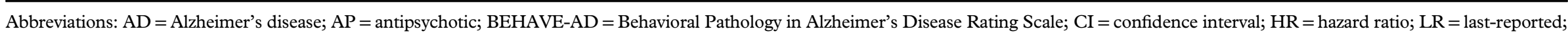

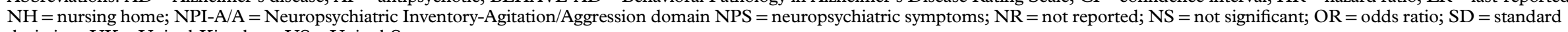
deviation; UK = United Kingdom; US = United States 
including 23 studies in the data synthesis. Overall, the authors concluded that NPS are highly prevalent and persistent, but frequency parameters varied considerably across studies. Data on agitation was provided by nine of the included studies, and agitation was noted as one of the symptoms, with high prevalence an increasing trend over time. Reported point prevalence rates for wandering or agitation were $18 \%$ to $62 \%$, while cumulative prevalence rate ranged between $40 \%$ to $100 \%$. These wide ranges are consistent with the findings from our review. Another point of consistency for our review is the large number of NPS measures used for assessment of NPS, which was also noted in a review by Wetzels and colleagues (2010a); this review identified 12 measures used across 18 publications.

A separate review examined the prevalence and course of NPS in patients with dementia, including population-based studies, outpatient, and long-term patient populations (Bergh and Selbæk, 2012). Only studies using the NPI were reviewed; prevalence rates were examined for persons with dementia included in population-based studies, attending outpatient clinics, or living in long-term care facilities. Agitation was among the symptoms with the highest prevalence rates reported - a median of $27 \%$ for population-based studies and long-term care patients, and $37 \%$ for outpatients. The course of agitation was persistent for outpatient populations, while resolution rates were reported for long-term facilities. On the other hand, Wetzels and colleagues (2010a) reported increasing trends for agitation, suggesting that population setting may be an important variable to consider when the course of agitation is examined.

Van Der Linde and colleagues (2012) conducted a systematic review to give a broad overview of the prevalence, course, biological and psychosocial associations, care, and outcomes of behavioral and psychological symptoms in an older population with dementia. The authors examined 36 reviews, but none focused specifically on agitation in AD. Agitation was noted as one of the most prevalent symptoms identified by reviews in people with dementia. Consistent with our results, the authors noted that behavioral and psychological prevalence vary widely across reviews, but no specifics on agitation prevalence rates were provided.

Findings from our review are aligned with previously reported findings on agitation prevalence and natural progression. This review concluded that information is particularly scarce in areas including incidence rates of agitation and studies specifically examining the relationship between agitation symptoms and mortality. No U.S. study on incidence rates was identified. A possible reason for the low number of studies on incidence of agitation is the challenge of defining, measuring, and differentiating agitation incidence versus agitation prevalence for a symptom that is not constantly present. Finally, information from studies conducted with community-dwelling patients is relatively scarce.

The results of this systematic review suggest a need to clearly establish a unified understanding of the commonalities surrounding agitation in the context of $\mathrm{AD}$, which should be built on a foundation of quality standardized methodology. Only by clearly understanding the incidence of agitation and its burden on $\mathrm{AD}$ patients can the medical community help identify the most appropriate treatment.

The key findings of this study need to be considered in the context of its limitations and study design. This systematic review was conceptualized as a broad review of information related to agitation burden for $\mathrm{AD}$, and all study designs were accepted. In addition, a definition of agitation consistent with the IPA definition was used, including specific behaviors and related NPS; this could have possibly introduced some noise in the key findings as there is variability in how agitation is defined and measured in the literature. The target population was also broadly defined as patients with $\mathrm{AD}$ or dementia. These factors may contribute to the wide variability of papers included in the systematic review and lead to challenges in consistently summarizing and interpreting all information, in addition to bringing inherent variability in results.

This review summarized available evidence on incidence, prevalence, and course of agitation, as well as studies on the association of agitation with $\mathrm{AD}$ severity and mortality. Consistent with earlier reports, agitation is highly prevalent, but the course of the symptoms over time seems to depend on multiple factors. Evidence for the association of agitation with $\mathrm{AD}$ appears to be strong, as most studies identified reported significant association despite heterogeneity in measures and designs used. There is limited information on the specific association of agitation symptoms and mortality; lack of existing evidence suggests additional studies to better understand possible associations is warranted.

\section{Conflict of interest}

Ruth A. Duffy, Ross A. Baker, and Myrlene Sanon Aigbogun are employed by Otsuka Pharmaceutical Development and Commercialization, Inc. Ann Hartry and Lene Hammer-Helmich are employed by Lundbeck LLC. Milena Anatchkova, Anne Brooks, and Laura Swett are employed by Evidera, which provides consulting and other research services to pharmaceutical, medical device, and related organizations. In their salaried positions, they work 
with a variety of companies and organizations, and are precluded from receiving payment or honoraria directly from these organizations for services rendered. Evidera received funding from Otsuka and Lundbeck to participate in the study and the development of this manuscript.

All authors participated in study design, data analysis and interpretation, and contributed to the development of the manuscript. All named authors meet the International Committee of Medical Journal Editors (ICMJE) criteria for authorship for this manuscript, take responsibility for the integrity of the work, and have given final approval to the version to be published.

\section{Description of authors' roles}

M. Anatchkova participated in the study design, supervised data collection and analyses, and drafted the paper. L. Swett and A. Brooks supervised data collection, conducted data analysis, and prepared tables for the manuscript. M. Sanon Aigbogun and A. Hartry participated in the study design, protocol review, review of analyses, interpretation of findings, and review of the manuscript. R. Duffy, R. Baker, and L. Hammer-Helmich participated in the protocol review, review of analyses, interpretation of findings, and review of the manuscript.

\section{Acknowledgments}

The authors thank Michael Grossi of the Evidera Editorial and Design Services team for his assistance in editing and preparing the manuscript for submission.

\section{Supplementary material}

To view supplementary material for this article, please visit https://doi.org/10.1017/S104161021800 1898.

\section{References}

Alzheimer's Association (2015). 2015 Alzheimer's disease facts and figures. Alzheimer's \& Dementia, 11, 332-384.

Alzheimer's Society (2011). Optimising Treatment and Care for People with Behavioural and Psychological Symptoms of Dementia. A Best Practice Guide for Health and Social Care Professionals. London: Alzheimer's Society.

Amoo, G. et al. (2011). Profile of clinically-diagnosed dementias in a neuropsychiatric practice in Abeokuta, south-western Nigeria. African fournal of Psychiatry, 14, 377-382. doi: 10.4314/ajpsy.v14i5.5.
Anatchkova, M. et al. (2017). The economic burden of agitation in Alzheimer's disease: a systematic literature review. Presented at ISPOR 22nd Annual International Meeting, May 20-24, Boston, MA.

Bergh, S., Engedal, K., Roen, I. and Selbaek, G. (2011). The course of neuropsychiatric symptoms in patients with dementia in Norwegian nursing homes. International Psychogeriatrics, 23, 1231-1239. doi: 10.1017/ S1041610211001177.

Bergh, S. and Selbæk, G. (2012). The prevalence and the course of neuropsychiatric symptoms in patients with dementia. Norsk Epidemiologi, 22, 225-232. doi: 10.5324/ nje.v22i2.1570.

Borsje, P., Wetzels, R. B., Lucassen, P. L., Pot, A. M. and Koopmans, R. T. (2015). The course of neuropsychiatric symptoms in community-dwelling patients with dementia: a systematic review. International Psychogeriatrics, 27, 385-405. doi: 10.1017/S1041610214002282.

Brodaty, H., Connors, M. H., Xu, J., Woodward, M., Ames, D. and PRIME Study Group (2015). The course of neuropsychiatric symptoms in dementia: a 3-year longitudinal study. Fournal of the American Medical Directors Association, 16, 380-387. doi: 10.1016/j.jamda .2014.12.018.

Burgio, L. D., Park, N. S., Hardin, J. M. and Sun, F. (2007). A longitudinal examination of agitation and resident characteristics in the nursing home. The Gerontologist, 47, 642-649. doi: 10.1093/geront/47.5.642.

Conde-Sala, J. L. et al. (2016). Effects of anosognosia and neuropsychiatric symptoms on the quality of life of patients with Alzheimer's disease: a 24-month follow-up study. International Fournal of Geriatric Psychiatry, 31, 109-119. doi: $10.1002 /$ gps.4298.

Cummings, J. et al. (2015). Agitation in cognitive disorders: International Psychogeriatric Association provisional consensus clinical and research definition. International Psychogeriatrics, 27, 7-17. doi: 10.1017/S1041610214001963.

D'Onofrio, G. et al. (2012). Neuropsychiatric symptoms and functional status in Alzheimer's disease and vascular dementia patients. Current Alzheimer Research, 9, 759-771. doi: $10.2174 / 156720512801322582$.

D'Onofrio, G. et al. (2016). Delusions in patients with Alzheimer's disease: a multidimensional approach. fournal of Alzheimer's Disease, 51, 427-437. doi: 10.3233/JAD150944.

de Oliveira, F. F., Wajman, J. R., Bertolucci, P. H., Chen, E. S. and Smith, M. C. (2015). Correlations among cognitive and behavioural assessments in patients with dementia due to Alzheimer's disease. Clinical Neurology and Neurosurgery, 135, 27-33. doi: 10.1016/j.clineuro .2015.05.010.

Fauth, E. B., Zarit, S. H., Femia, E. E., Hofer, S. M. and Stephens, M. A. (2006). Behavioral and psychological symptoms of dementia and caregivers' stress appraisals: intra-individual stability and change over short-term observations. Aging and Mental Health, 10, 563-573. doi: 10.1080/13607860600638107.

Fernandez-Martinez, M., Molano, A., Castro, J. and Zarranz, J. J. (2010). Prevalence of neuropsychiatric symptoms in mild cognitive impairment and Alzheimer's disease, and its relationship with cognitive impairment. Current Alzheimer Research, 7, 517-526. 
Fernandez Martinez, M., Flores, J. C., De Las Heras, S. P., Lekumberri, A. M., Menocal, M. G. and Imirizaldu, J. J. Z. (2008). Prevalence of neuropsychiatric symptoms in elderly patients with dementia in Mungialde County (Basque Country, Spain). Dementia and Geriatric Cognitive Disorders, 25, 103-108. doi: 10.1159/000112215.

Gonfrier, S., Andrieu, S., Renaud, D., Vellas, B. and Robert, P. H. (2012). Course of neuropsychiatric symptoms during a 4-year follow up in the REAL-FR cohort. The fournal of Nutrition, Health and Aging, 16, 134-137.

Gustafsson, M., Sandman, P. O., Karlsson, S., Gustafson, Y. and Lovheim, H. (2013). Association between behavioral and psychological symptoms and psychotropic drug use among old people with cognitive impairment living in geriatric care settings. International Psychogeriatrics, 25, 1415-1423. doi: 10.1017/ S1041610213000859.

Hamuro, A. et al. (2007). Behavioral and psychological symptoms of dementia in untreated Alzheimer's disease patients. Psychogeriatrics, 7, 4-7. doi: 10.1111/j.1479-8301 .2006.00153.x.

Helvik, A. S. et al. (2016). Severity of neuropsychiatric symptoms in nursing home residents. Dementia and Geriatric Cognitive Disorders Extra, 6, 28-42. doi: 10.1159/ 000442250.

Hendriks, S. A., Smalbrugge, M., Galindo-Garre, F., Hertogh, C. M. and van der Steen, J. T. (2015). From admission to death: prevalence and course of pain, agitation, and shortness of breath, and treatment of these symptoms in nursing home residents with dementia. Fournal of the American Medical Directors Association, 16, 475-481. doi: 10.1016/j.jamda.2014.12.016.

Huang, T. Y., Wei, Y. J., Moyo, P., Harris, I., Lucas, J. A. and Simoni-Wastila, L. (2015). Treated behavioral symptoms and mortality in medicare beneficiaries in nursing homes with Alzheimer's disease and related dementias. Fournal of the American Geriatrics Society, 63, 1757-1765. doi: 10.1111/jgs.13606.

Ismail, M. S., Dagerman, K., Tariot, P. N., Abbott, S., Kavanagh, S. and Schneider, L. S. (2007). National Institute of Mental Health Clinical Antipsychotic Trials of Intervention Effectiveness- Alzheimer's Disease (CATIEAD): baseline characteristics. Current Alzheimer Research, 4, 325-335.

Karttunen, K. et al. (2011). Neuropsychiatric symptoms and quality of life in patients with very mild and mild Alzheimer's disease. International fournal of Geriatric Psychiatry, 26, 473-482. doi: 10.1002/gps.2550.

Kasai, M., Meguro, K., Akanuma, K. and Yamaguchi, S. (2015). Alzheimer's disease patients institutionalized in group homes run by long-term care insurance exhibit fewer symptoms of behavioural problems as evaluated by the Behavioural Pathology in Alzheimer's Disease Rating Scale. Psychogeriatrics, 15, 102-108.

Khoo, S. A., Chen, T. Y., Ang, Y. H. and Yap, P. (2013). The impact of neuropsychiatric symptoms on caregiver distress and quality of life in persons with dementia in an Asian tertiary hospital memory clinic. International Psychogeriatrics, 25, 1991-1999. doi: 10.1017/ S1041610213001518.
Kim, H. and Lee, K. J. (2014). Serum homocysteine levels are correlated with behavioral and psychological symptoms of Alzheimer's disease. Neuropsychiatric Disease and Treatment, 10, 1887-1896. doi: 10.2147/NDT.S68980.

Kitamura, T., Kitamura, M., Hino, S., Tanaka, N. and Kurata, K. (2012). Gender differences in clinical manifestations and outcomes among hospitalized patients with behavioral and psychological symptoms of dementia. Fournal of Clinical Psychiatry, 73, 1548-1554. doi: 10.4088/ JCP.11m07614.

Koppitz, A., Bosshard, G., Schuster, D. H., Hediger, H. and Imhof, L. (2015). Type and course of symptoms demonstrated in the terminal and dying phases by people with dementia in nursing homes. Zeitschrift Für Gerontologie und Geriatrie, 48, 176-183. doi: 10.1007/s00391-0140668-z.

Koyama, A., Fujise, N., Matsushita, M., Ishikawa, T., Hashimoto, M. and Ikeda, M. (2015). Suicidal ideation and related factors among dementia patients. Fournal of Affective Disorders, 178, 66-70. doi: 10.1016/j.jad.2015.02 .019 .

Liu, C. Y., Wang, P. N., Lin, K. N. and Liu, H. C. (2007). Behavioral and psychological symptoms in Taiwanese patients with Alzheimer's disease. International Psychogeriatrics, 19, 605-613. doi: 10.1017/ S1041610207005121.

Lustenberger, I., Schupbach, B., von Gunten, A. and Mosimann, U. (2011). Psychotropic medication use in Swiss nursing homes. Swiss Med Wkly, 141, w13254. doi: $10.4414 / \mathrm{smw} .2011 .13254$.

Mitchell, S. L. et al. (2009). The clinical course of advanced dementia. New England fournal of Medicine, 361, 1529-1538. doi: 10.1056/NEJMoa0902234.

Morgan, R. O., Sail, K. R., Snow, A. L., Davila, J. A., Fouladi, N. N. and Kunik, M. E. (2013). Modeling causes of aggressive behavior in patients with dementia. The Gerontologist, 53, 738-747. doi: 10.1093/geront/gns 129.

Mulders, A. J., Fick, I. W., Bor, H., Verhey, F. R., Zuidema, S. U. and Koopmans, R. T. (2016). Prevalence and correlates of neuropsychiatric symptoms in nursing home patients with young-onset dementia: the BEYOnD study. Fournal of the American Medical Directors Association, 17, 495-500. doi: 10.1016/j.jamda.2016.01.002.

O'Donnell, M. J., Lewis, D. L., Dubois, S., Standish, T. I., Bédard, M. and Molloy, D. W. (2007). Behavioural and psychological symptoms in communitydwelling elderly persons with cognitive impairment and dementia: prevalence and factor analysis. Clinical Gerontologist, 30, 41-52.

Orengo, C. A. et al. (2008). Aggression in individuals newly diagnosed with dementia. American fournal of Alzheimer's Disease E Other Dementias, 23, 227-232. doi: 10.1177/ 1533317507313373.

Pan, W. D. et al. (2013). Quantitative evaluation of severity of behavioral and psychological symptoms of dementia in patients with vascular dementia. Translational Neurodegeneration, 2, 9. doi: 10.1186/2047-9158-2-9.

Park, H. K. et al. (2015). Cognitive profiles and neuropsychiatric symptoms in Korean early-onset Alzheimer's disease patients: a CREDOS study. Fournal of Alzheimer's Disease, 44, 661-673. doi: 10.3233/JAD-141011. 
Peters, M. E. et al. (2015). Neuropsychiatric symptoms as predictors of progression to severe Alzheimer's dementia and death: the Cache County Dementia Progression Study. American fournal of Psychiatry, 172, 460-465. doi: 10.1176/appi.ajp.2014.14040480.

Pinidbunjerdkool, A., Saengwanitch, S. and Sithinamsuwan, P. (2014). Behavioral and psychological symptoms of dementia. Fournal of the Medical Association of Thailand, 97 Suppl 2, S168-S174.

Pink, A. et al. (2015). Neuropsychiatric symptoms, APOE epsilon4, and the risk of incident dementia: a populationbased study. Neurology, 84, 935-943. doi: 10.1212/WNL .0000000000001307 .

Porsteinsson, A. P. et al. (2014). Effect of citalopram on agitation in Alzheimer disease: the CitAD randomized clinical trial. $\mathcal{F} A M A, 311,682-691$. doi: 10.1001/jama .2014 .93 .

Sampson, E. L. et al. (2014). Behavioural and psychiatric symptoms in people with dementia admitted to the acute hospital: prospective cohort study. The British fournal of Psychiatry, 205, 189-196. doi: 10.1192/bjp.bp.113 .130948 .

Sampson, E. L. et al. (2015). Pain, agitation, and behavioural problems in people with dementia admitted to general hospital wards: a longitudinal cohort study. Pain, 156, 675-683. doi: 10.1097/j.pain.00000000 00000095.

Scarmeas, N. et al. (2007). Disruptive behavior as a predictor in Alzheimer disease. Archives of Neurology, 64, 1755-1761. doi: 10.1001/archneur.64.12.1755.

Selbaek, G., Engedal, K., Benth, J. S., Bergh, S. (2014). The course of neuropsychiatric symptoms in nursing-home patients with dementia over a 53-month follow-up period. International Psychogeriatrics, 26, 81-91. doi: 10.1017/ S1041610213001609.

Selbaek, G., Kirkevold, O. and Engedal, K. (2008). The course of psychiatric and behavioral symptoms and the use of psychotropic medication in patients with dementia in Norwegian nursing homes-a 12-month follow-up study. The American fournal of Geriatric Psychiatry, 16, 528-536. doi: 10.1097/JGP.0b013e318167ae76.

Spalletta, G. et al. (2010). Neuropsychiatric symptoms and syndromes in a large cohort of newly diagnosed, untreated patients with Alzheimer disease. The American fournal of Geriatric Psychiatry, 18, 1026-1035. doi: 10.1097/JGP $.0 \mathrm{~b} 013 \mathrm{e} 3181 \mathrm{~d} 6 \mathrm{~b} 68 \mathrm{~d}$.

Steinberg, M. et al. (2006). Risk factors for neuropsychiatric symptoms in dementia: the Cache County Study. International fournal of Geriatric Psychiatry, 21, 824-830. doi: $10.1002 /$ gps.1567.

Steinberg, M. et al. (2008). Point and 5-year period prevalence of neuropsychiatric symptoms in dementia: the Cache County Study. International fournal of Geriatric Psychiatry, 23, 170-177. doi: 10.1002/gps.1858.
Stella, F., Laks, J., Govone, J. S., de Medeiros, K. and Forlenza, O. V. (2016). Association of neuropsychiatric syndromes with global clinical deterioration in Alzheimer's disease patients. International Psychogeriatrics, 28, 779-786. doi: $10.1017 /$ S1041610215002069.

Tanaka, H. et al. (2015). Relationship between dementia severity and behavioural and psychological symptoms in early-onset Alzheimer's disease. Psychogeriatrics, 15, 242-247. doi: 10.1111/psyg.12108.

Treiber, K. A. et al. (2008). Vascular factors and risk for neuropsychiatric symptoms in Alzheimer's disease: the Cache County Study. International Psychogeriatrics, 20, 538-553. doi: 10.1017/S1041610208006704.

Trzepacz, P. T. et al. (2013). Frontolimbic atrophy is associated with agitation and aggression in mild cognitive impairment and Alzheimer's disease. Alzheimer's \& Dementia, 9, S95-S104 e1. doi: 10.1016/j.jalz.2012.10.005.

van der Linde, R. M., Stephan, B. C., Savva, G. M., Dening, T. and Brayne, C. (2012). Systematic reviews on behavioural and psychological symptoms in the older or demented population. Alzheimer's Research \& Therapy, 4, 28. doi: 10.1186/alzrt131.

Vandervoort, A. et al. (2013). Nursing home residents dying with dementia in Flanders, Belgium: a nationwide postmortem study on clinical characteristics and quality of dying. Fournal of the American Medical Directors Association, 14, 485-492. doi: 10.1016/j.jamda.2013.01.016.

Vogel, A., Waldorff, F. B. and Waldemar, G. (2015). Longitudinal changes in awareness over 36 months in patients with mild Alzheimer's disease. International Psychogeriatrics, 27, 95-102. doi: 10.1017/S10416102 14001562.

Wetzels, R. B., Zuidema, S. U., de Jonghe, J. F., Verhey, F. R. and Koopmans, R. T. (2010a). Course of neuropsychiatric symptoms in residents with dementia in nursing homes over 2-year period. The American fournal of Geriatric Psychiatry, 18, 1054-1065.

Wetzels, R. B., Zuidema, S. U., de Jonghe, J. F., Verhey, F. R. and Koopmans, R. T. (2010b). Determinants of quality of life in nursing home residents with dementia. Dementia and Geriatric Cognitive Disorders, 29, 189-197. doi: 10.1159/000280437.

Wolf-Ostermann, K., Worch, A., Fischer, T., Wulff, I. and Graske, J. (2012). Health outcomes and quality of life of residents of shared-housing arrangements compared to residents of special care units - results of the Berlin DeWeGE-study. Fournal of Clinical Nursing, 21, 3047-3060. doi: 10.1111/j.1365-2702.2012.04305.x.

Zahodne, L. B., Ornstein, K., Cosentino, S., Devanand, D. P. and Stern, Y. (2015). Longitudinal relationships between Alzheimer disease progression and psychosis, depressed mood, and agitation/aggression. The American Fournal of Geriatric Psychiatry, 23, 130-140. doi: 10.1016/j. jagp.2013.03.014. 